\title{
Cluster based Efficient File Replica in P2P Mobile Ad Hoc Network
}

\author{
R.Lakshmi Prabha \\ PG Student \\ Department of Computer Science \\ K.L.N. College of Information \& Technology \\ Madurai, India
}

\author{
G.Ganesan \\ Associate Professor \\ Department of Computer Science \\ K.L.N. College of Information \& Technology \\ Madurai, India
}

\begin{abstract}
Mobile Ad hoc Networks (MANET) consists of collection of wireless mobile devices dynamically forming a temporary network without the use of any existing infrastructure or centralized administration. Based on Peer-to-Peer (P2P) network, file sharing is mainly established in MANET. Design and implementation of secured cooperative cache in wireless P2P networks are presented, and proposed solutions to detect the best place to cache the data. The goal of work is to improve file availability for effective file sharing in peer to peer mobile ad hoc network. And also we make analysis of distributed routing protocol DSR and AODV in dynamic peer to peer mobile ad hoc network (MANET) with influence of through gateway transmission.
\end{abstract}

\section{Keywords}

MANET, peer to peer network, File sharing, cooperative cache.

\section{INTRODUCTION}

In a mobile ad hoc network (MANET), mobile hosts can communicate directly with one another using direct pair wireless links. Because it requires no fixed infrastructure and most of the time no explicit administration a MANET can extremely useful to support communication in challenging situations, such as in rural, remote, or disaster-struck areas. $\mathrm{P} 2 \mathrm{P}$ computing refers to technology that enables two or more peers to collaborate spontaneously in a network of equals (peers) by using appropriate information and communication systems without the necessity for central coordination.

P2P networks are overlay networks typically operated on infrastructure (wired) networks, such as the Internet. However, the P2P overlay network is dynamic, where peers come and go (i.e., leave and join the group) for sharing files and data through direct exchange. Such peer-to-peer communication paradigm will be very important in wireless multi-hop networks as centralized servers might not be available or located in the Internet. Therefore, P2P will be an interesting alternative for decentralizing services or making its own local resources available in the multi-hop network to serve local user communities. P2P overlay networks in the Internet and mobile ad-hoc networks share many key characteristics such as self-organization and decentralization due to the common nature of their distributed components.

\section{EXISTING SYSTEM}

The efficiency of file querying suffers from the distinctive properties of mobile ad hoc networks including node mobility and limited communication range and resource.
Existing ad hoc network mainly focused on global optimal replica creation with minimum average querying delay. New concept of resource for which considers both node storage and meeting frequency. File replication is an effective way to enhance file availability and reduce file querying delay. It creates replicas for a file to improve its probability of being encountered by request. Each individual node replicates files it frequently queries or a group of nodes create one replica for each file they frequently query. Unfortunately, it is impractical and inefficient to enable every node to hold the replicas of all files in the system considering limited node resource. Though redundant replicas are reduced by group cooperation, neighbouring nodes may separate from each other due to node mobility, leading to large query delay.

\section{Limitations on Existing System}

- Leads to large query delay due to node mobility.

- Each individual node creates one replica it frequently queries, which cause waste of resources.

- Mobility of node affects the availability of files or message.

\section{PROPOSED SYSTEM}

File sharing in peer to peer mobile ad hoc network is made efficient and reduced delay with help increased cache size. In file sharing, it looks for two operation,

\section{- Cache miss \\ - Cache penalty}

A cache miss refers to a failed attempt to read or write a piece of data in the cache, which results main memory access takes longer latency. Cache misses can be of three types: data read miss, instruction read miss and data write miss.

Design and implementation of secured cooperative cache in wireless P2P networks are presented. Through real implementations, important design issues are identified and proposed an asymmetric approach in order to reduce the overhead of copying data between the user space and the kernel space, and also to reduce the data processing delay.

The proposed algorithm well considers the caching overhead and adapts the cache node selection strategy to maximize the caching benefit on different MAC layers. Results show that the asymmetric approach outperforms the symmetric approach in traditional 802.11- based ad hoc networks due to removal of most of the processing overhead. 


\section{IMPLEMENTATION}

\subsection{System Design}

Architectural diagram deals with cluster of mobile nodes. In this architecture each cluster contains cluster head. Each cluster head of each cluster is communicated with centralized node. Cluster head is also elected by centralized node. Each member in the cluster is identical in some features. Each member in the cluster requests the cluster head for files and data. Cluster head includes all information about cluster members. Cluster head one group must communicate to cluster head of another cluster in order to get information of cluster member of that particular cluster.

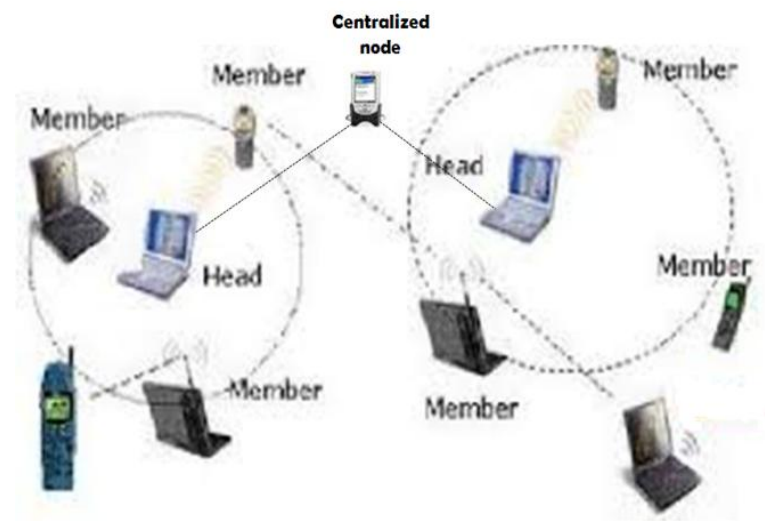

Fig 1: MANET Architecture

\subsection{Modules Description}

\subsubsection{Cache and routing module \\ Cell Creation:}

Cells are created with default $\mathrm{X}$ and $\mathrm{Y}$ axis. Each cell has a unique Id and position of each cell is displayed with help of $\mathrm{x}$, y points. All cells have a constant size which can be declared previously. Each cell have adjacent cells and particularly with maximum of six adjacent cells. Adjacency of each cell is always specified in adjacency matrix.

\section{Node Insertion}

Each cell can have multiple numbers of nodes. Node count for each cell has to be specified initially. Nodes are specified in a position with $\mathrm{x}, \mathrm{y}$ points. Nodes in a network are movable since it is a mobile ad hoc network.

\section{Gateway selection}

Each cell have multiple number of nodes within that gateway node is selected. Gateway is a router or a proxy server that routes between networks. In this, gateway node acts as router between the cells. Always node which is near to the required adjacency cell is selected as a gateway node in order to reach destination with reduced time and delay. .

\subsubsection{Cache routing simulation module: Path finding:}

Before finding the path between nodes. Source node and destination node are selected. With help of dynamic routing protocol DSR or AODV, efficient path can be established through gateway of each cell. Gateway could find exact direction of destination node. Use of dynamic routing protocol, whether DSR or AODV is previous selected. With that, distance, hope count, time duration can be found.

\subsubsection{Caching and Routing module}

\section{Authentication:}

Diffie Hellman Digital Signature scheme is used as authentication technique in peer to peer mobile ad hoc network. Digital signature is mainly used for demonstrating the authenticity of the digital message or document. Digital signature is performed by a signing algorithm and it is verified by a verification algorithm.

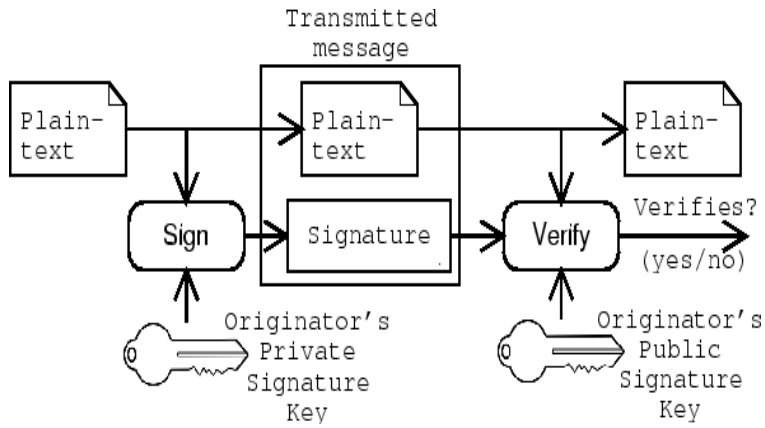

Fig 2: Digital Signature

Here authenticity is verified for checking whether respective gateway node or any other node is active. Authenticity also verifies for correct routing path, if the path does not reach the destination reports a unsuccessful result.

\section{IMPLEMENTATION RESULT}

\begin{tabular}{|l|l|l|l|l|l|l|l|}
\multicolumn{3}{c|}{ PATH } & \multicolumn{3}{c|}{ DSR } & \multicolumn{3}{c|}{ AODV } \\
\hline $\begin{array}{l}\text { SOURCE } \\
\text { NODE }\end{array}$ & $\begin{array}{l}\text { DESTINATION } \\
\text { NODE }\end{array}$ & $\begin{array}{l}\text { HOP } \\
\text { COUNT }\end{array}$ & DISTANCE & TIME & $\begin{array}{l}\text { HOP } \\
\text { COUNT }\end{array}$ & DISTANCE & TIME \\
\hline 10 & 25 & 3 & 109 & 1.64 & 1 & 47 & 0.844 \\
\hline 50 & 70 & 4 & 141 & 2.062 & 2 & 83 & 1.234 \\
\hline 80 & 90 & 8 & 247 & 3.672 & 4 & 134 & 2.047 \\
\hline 40 & 90 & 6 & 230 & 2.875 & 4 & 276 & 3.688 \\
\hline 80 & 150 & 10 & 350 & 4.5 & 9 & 293 & 4.094 \\
\hline 100 & 170 & 19 & 623 & 8.141 & 11 & 414 & 4.891 \\
\hline
\end{tabular}

Fig 3: Analysis of DSR and AODV

Analysis Report of distributed routing protocol DSR and AODV represented in a tabular format. Report deals with different ranges of source and destination node values. Ranges will be of (1-50),(1-100),(1-150),(1-200). For each certain sample values are taken. Example for 1-50 range source node value is 10 and destination node value is 25 which are between range1-50. Likewise same procedure repeated for other ranges and analysis of time, distance, hop count is made. Variation in values corresponding to Time taken, Distance, and hop count is noted. According to that simple graphical representation is made with respect to distance and time. 


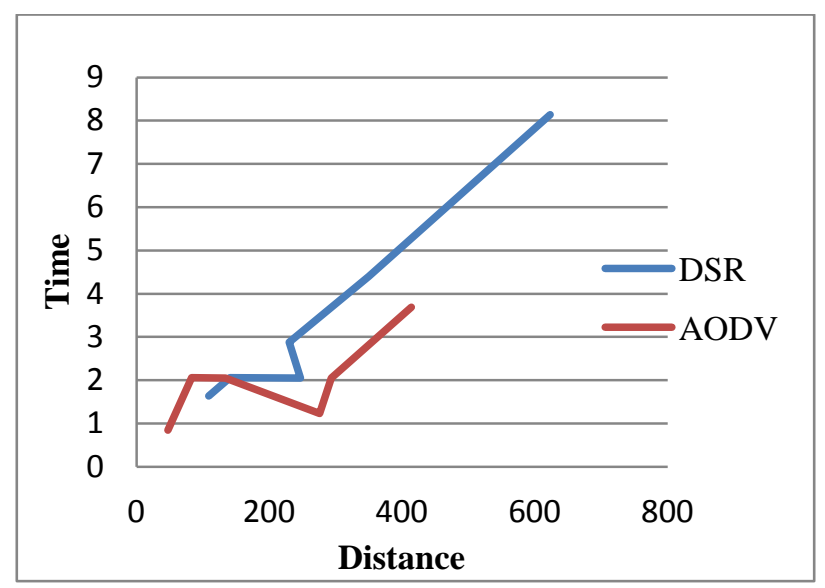

Fig 4: Distance vs Time

Graph discuss with analysis of distributed routing protocol DSR and AODV. The graph deals with the comparison of routing protocol for same source and destination values. Time taken, distance, hop count values are gradually high for DSR comparing to AODV routing protocol. Analysis Result is shown in the Graph.

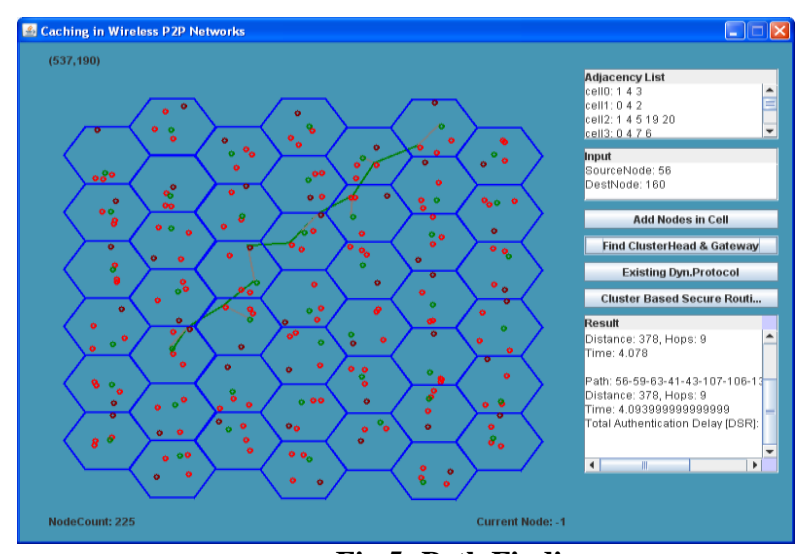

Fig 5: Path Finding

Analysis of distributed routing protocol DSR and AODV in $\mathrm{p} 2 \mathrm{p}$ mobile ad hoc network is analysed in implementation test bed Net Beans IDE 8.0.1. And final output representation is shown.

\section{CONCLUSION AND FUTURE ENHANCEMENT}

Dynamic routing protocol DSR and AODV performance is enhanced in peer to peer mobile ad hoc network using gateway node and cooperative caching. Authenticated routing path is established from source to destination in peer to peer mobile ad hoc network with help of Diffie Hellman digital signature scheme.

Our future work will concentrate on efficient and secured file sharing in peer to peer mobile ad hoc network by implementing the proposed technique improved Euclidean algorithm.

\section{REFERENCES}

[1] Kang Chen and Haiying Shen, "Maximizing P2P File Access Availability in Mobile Ad hoc Networks Though Replication for Efficient File Sharing",2014.

[2] T.H.Feiroz khan, "On maximizing data accessibility in mobile ad hoc network", Vol. 5 No. 04S Apr 2013.

[3] Z. Li and H. Shen, "Sedum: Exploiting social networks in utility based distributed routing for DTNs," TC, 2012.

[4] Z. Li and H. Shen, "Analysis of cooperation incentive strategies in mobile ad hoc networks," TMC, 2012.

[5] X. Zhuo, Q. Li, G. Cao, Y. Dai, B. K. Szymanski, and T. L. Porta,"Social-based cooperative caching in DTNs: A contact duration aware approach." in Proc. of MASS, 2011.

[6] W. Gao, G. Cao, A. Iyengar, and M. Srivatsa, "Supporting cooperative caching in disruption tolerant networks." in Proc. of ICDCS,2011.

[7] S. Ioannidis, L. Massoulie, and A. Chaintreau, "Distributed caching over heterogeneous mobile networks." in Proc. of SIGMETRICS, 2010. 